\title{
Resonancia magnética en trauma craneal moderado y grave: estudio comparativo de hallazgos en TC y RM. Características relacionadas con la presencia y localización de lesión axonal difusa en RM
}

\author{
A. Lagares; A. Ramos*; R. Alday; F. Ballenilla*; A. Pérez-Núñez; I. Arrese; J.F. Alén; B. Pascual; A. Kaen; P.A. \\ Gómez y R.D. Lobato \\ Servicios de Neurocirugía y Neurorradiología*. Hospital 12 de Octubre. Madrid.
}

Resumen

Introducción. La TC craneal ha sido el método más extendido en la evaluación de enfermos que han sufrido trauma craneal. Sin embargo, es poco sensible en la identificación de lesión axonal difusa y lesiones en fosa posterior. La RM craneal es una prueba potencialmente más sensible pero de difícil realización en estos enfermos, hecho que ha impedido la generalización de su uso.

Objetivos. Comparar la capacidad de identificación de lesiones intracraneales postraumáticas por parte de las dos pruebas diagnósticas en enfermos con TCE grave y moderado, y determinar qué características radiológicas en la $\mathrm{TC}$ se asocian a la presencia de $\mathrm{LAD}$ en RM y su gravedad clínica.

Material y métodos. Se incluyen en el estudio 100 enfermos con TCE moderado y grave a los que se ha realizado RM craneal dentro de los primeros 30 días tras el trauma craneal. Se recogieron todas las variables clínicas potencialmente relacionadas con el pronóstico de los enfermos, así como los datos del TC inicial según la clasificación de Marshall y cols. La RM fue evaluada de manera ciega por dos neurorradiólogos que ignoraban al resultado de la TC inicial y la situación clínica inicial del paciente. Se recogieron todas las lesiones que presentaban, así como su clasificación según la clasificación de lesiones asociadas con LAD, descrita por Adams. Se compararon los hallazgos en TC y RM, evaluando la sensibilidad de cada prueba con respecto a los diferentes hallazgos. Se estudiaron los hallazgos relacionados con la presencia de $L A D$ en $R M$, mediante estudio univariable, usando la prueba de $\chi^{2}$ y correlaciones simples.

Resultados. La RM es más sensible que la TC para las lesiones en sustancia blanca cerebral, cuerpo calloso y tronco. Además, detecta mayor número de contusiones. La presencia de lesión axonal difusa depende del mecanismo de producción del trauma, siendo más

Recibido: 12-05-05. Aceptado: 31-05-05 frecuente en traumas de mayor energía, sobre todo en los accidentes de tráfico, bien sea con automóvil o moto/bici. En cuanto a las características radiológicas asociadas a LAD la más claramente relacionada es la hemorragia intraventricular. La presencia de daño cada vez más profundo y mayor puntuación en la escala de Adams se asocia a menor puntuación en la GCS y GCS motora, y por consiguiente peor nivel de conciencia y mayor gravedad del trauma inicial, confirmando el modelo de Ommaya.

PALABRAS CLAVE: Lesión traumática cerebral. Trauma craneal. RM. TC. Lesión axonal difusa. Métodos de imagen

Magnetic Resonance in moderate and severe head injury: comparative study of CT and MR findings. Characteristics related to the presence and location of diffuse axonal injury in MR

Summary

Introduction. Cranial CT has been the most extended evaluation means for patients suffering head trauma. However, it has low sensitivity in the identification of diffuse axonal injury and posterior fossa lesions. Cranial MR is a potentially more sensitive test but difficult to perform in these patients, a fact that has hampered its generalised use.

Objective. To compare the identification capability of traumatic intracranial lesions by both diagnostic tests in patients with moderate and severe head injury and to determine which radiological characteristics are associated with the presence of diffuse injury in MR and their clinical severity.

Material and methods. 100 patients suffering moderate or severe head injury to whom a MR had been performed in the first 30 days after trauma were included. All clinical variables related to prognosis were registered, as well as the data from the initial CT following Marshall et al., classification. The MR was blindly 
evaluated by two neuroradiologists that were not aware of the initial CT results or the clinical situation of the patient. All lesions were registered as well as the classification following the classification of lesions related to DAI described by Adams et al. CT and MR findings were compared evaluating the sensitivities of each test. Factors related to the presence of diffuse injury in MR were studied by univariate analysis using $\chi^{2}$ test and simple correlations.

Results. MR is more sensitive than CT for lesions in cerebral white matter, corpus callosum and brainstem. It also detects a greater number of cerebral contussions. The presence of diffuse axonal injury depends on the mechanism of the trauma, being more frequent in higher energy trauma, specially in traffic accidents. Among the radiological characteristics associated to DAI the most clearly related is intraventricular haemorrhage. The presence of a deeper injury and a higher score in the scales of Adams is associated with a lower score in the GCS and motor GCS, and so with a worse level of consciousness and bigger severity of injury, confirming Ommaya's model.

KEY WORDS: Traumatic brain injury. Head trauma. MR. CT. Diffuse axonal injury. Imaging methods.

\section{Introducción}

El traumatismo craneoencefálico (TCE) es una de las causas principales de discapacidad neurológica que afecta, fundamentalmente, a pacientes jóvenes ${ }^{4,5}$. En las últimas décadas la TC craneal ha sido la técnica más utilizada en el diagnóstico de las lesiones que presentaban estos enfermos y ha colaborado en el mejor conocimiento de la fisiopatología del TCE y en su mejor manejo terapéutico ${ }^{20,25}$. Sin embargo, es bien conocido el hecho de que muchos enfermos presentan alteraciones importantes del nivel de conciencia tras TCE y posteriormente presentan secuelas neurológicas secundarias al mismo, sin que presenten hallazgos relevantes en los TC realizados durante su evolución ${ }^{7,29}$. Además, la TC tiene una capacidad de resolución limitada en lesiones no hemorrágicas y en las localizadas en la fosa posterior.

La RM craneal surgió como una prueba diagnóstica muy sensible a lesiones de tipo no hemorrágico y en fosa posterior y, por ello, podría tener un papel en el diagnóstico de las lesiones intracraneales postraumáticas ${ }^{10,12,31}$. Sin embargo, los mayores tiempos requeridos para realizar la exploración con este método diagnóstico y las dificultades técnicas en su realización en enfermos clínicamente inestables, ha hecho que su utilización de forma generalizada en el diagnóstico del TCE sea excepcional y limitada a un número pequeño de centros. En la actualidad se han aña- dido diversas secuencias que requieren menores tiempos de exploración, hecho que ha facilitado el incremento de su utilización.

Según diversos autores, la lesión axonal difusa (LAD) es en gran medida responsable de la morbilidad y mortalidad asociada al TCE grave $e^{1,2,15,16,25}$. Se han propuesto diversas teorías para explicar su aparición, pero el modelo fisiopatológico más aceptado es el propuesto por Ommaya y Gennarelli, en relación a hallazgos neuropatológicos, en el que, a medida que aumenta la intensidad del trauma, los hallazgos de lesiones se sitúan más profundamente, desde la corteza hasta el tronco cerebral, y que esta gradación en profundidad estaría en relación al deterioro de conciencia que el enfermo presenta9. La RM craneal, al ser capaz de detectar con mayor sensibilidad las lesiones relacionadas con $\mathrm{LAD}$, podría ser útil a la hora de establecer si este modelo fisiopatológico es correcto.

El objetivo del presente trabajo es doble. En primer lugar, se comparará la capacidad de identificación de lesiones intracraneales postraumáticas por parte de las dos pruebas diagnósticas más útiles, esto es la TC y RM, en enfermos con TCE grave y moderado. En segundo lugar, se determinarán qué características radiológicas en la TC se asocian a la presencia de LAD en RM y su gravedad clínica.

\section{Material y métodos}

\section{Criterios de inclusión}

En el presente estudio se han revisado los hallazgos clínicos y radiológicos en 100 enfermos con TCE grave y moderado estudiados con RM en el momento agudo-subagudo del TCE (primeros 30 días de evolución). Para ello se incluyeron en el estudio de forma prospectiva los enfermos aplicando los siguientes criterios de inclusión y exclusión:

- Criterios de inclusión:

- Edad entre 15 y 75 años.

- TCE grave/moderado (GCS postresucitación $<=$

12) ó GCS $=13$ además de pérdida de conocimiento y amnesia postraumática.

- Criterios de exclusión:

- Signos de muerte cerebral al ingreso (midriasis bilateral arreactiva, etc.).

- Imposibilidad para realizar TC precoz por inestabilidad hemodinámica importante $\mathrm{u}$ otros motivos.

- Muerte precoz en la etapa aguda-subaguda con imposibilidad de realizar RM.

- Imposibilidad de seguimiento posterior.

\section{Procedimientos:}

Todo paciente que ingresa en nuestro Hospital con un 


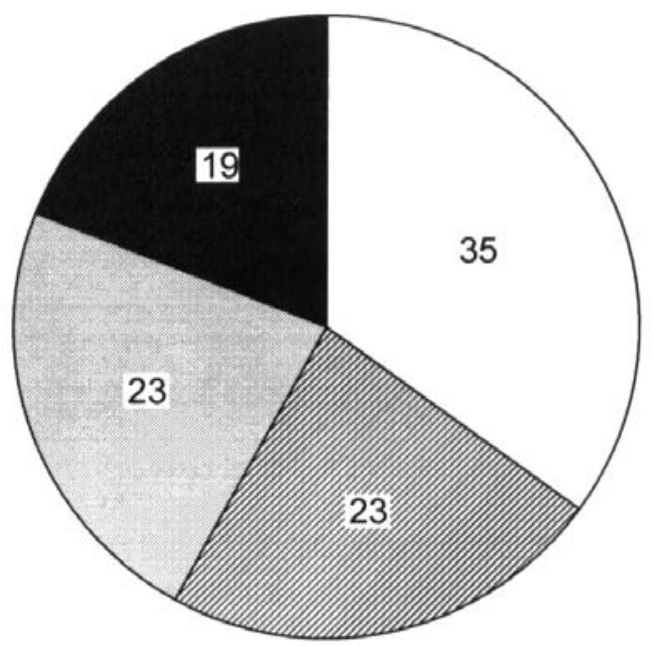

Clasificación según la

escala de Adams y cols.

$\square$ No LAD

Lesiones confinadas en sustancia

blanca de lóbulos frotales y

temporales

Lesiones en sustancia blanca

lobar y parte posterior cuerpo

calloso

- Lesiones dórsolaterales

mesencéfalo

Figura 1. Clasificación de los hallazgos en RM según la clasificación de Adams, mostrando los porcentajes que corresponden a cada grado de la clasificación.

TCE moderado o grave es valorado en la Unidad de Cuidados Intensivos de Politraumatizados. Tras su estabilización hemodinámica se practica una TC craneal. En los casos de TCE grave, si no existe efecto de masa en el TC inicial, se coloca un sensor de presión intracraneal y se manejan los pacientes según un protocolo estandarizado. Los pacientes con TCE moderado, dependiendo de las lesiones asociadas y las lesiones en TC, son tratados en la UCI o en la planta de Neurocirugía. En todos los casos, tras la TC inicial del ingreso, esta exploración se repite a las 12-24 y 36 horas del trauma. Esta pauta puede variar dependiendo de la evolución clínica y si la primera TC se realizase muy precozmente tras el TCE $(<3$ horas).

En los casos de TCE moderado, con pérdida de conciencia o amnesia, los pacientes son valorados inicialmente por el Servicio de Neurocirugía y estudiados con TC craneal. Posteriormente, y si no existen efectos de masa en la TC, quedan ingresados en la planta de Neurocirugía donde son vigilados neurológicamente y se les practican sucesivas TC de control dependiendo de la lesión en la TC inicial.

\section{Protocolo de imagen en RM}

A todos los enfermos que cumplían los criterios de inclusión se les realizó una RM lo más precozmente posible en su evolución siguiendo los siguientes parámetros técnicos.

- Sagital T1 Flair: TR: 2000; TE: MIN FULL (8-48); TI: 750; NEX: 2; Matriz: 256x256; VB: 31,25; Thickness: 5; Spacing: 1; Time 2,58.

- Axial Flair: TR: 10000; TE: 145; TI: 2200; NEX: 1; VB: 20; Matriz: 256x192; Thickness: 5; Spacing:1; Time:4.

- Axial T2: TR: 4000; TE: 85; ETL: 12; VB: 20; Matriz: 384x256; NEX: 2; Thickness: 5; Spacing: 1; Time: 2,16.

- Axial gradiente T2: TR: 550; TE: 18; FLIP ANGLE: 28; VB: 15; Matriz: 256x224; NEX: 2; Thickness: 5; Spacing: 1 ; Time :3,04.

\section{Recogida de datos}

Al ingreso se recogieron datos epidemiológicos tales como edad, sexo, mecanismo del TCE, presencia de trauma extracraneal grave asociado, nivel de conciencia postresucitación, según la escala de coma de Glasgow (GCS) y su subescala motora, y estado pupilar. Así mismo, se recogieron los hallazgos en la TC inicial expresados según la escala del Traumatic Coma Data Bank que clasifica los hallazgos en imagen según la presencia o ausencia de lesiones masa, compresión cisternal y/o desplazamiento de línea media ${ }^{19}$.

Además, se identificaron y registraron las diferentes lesiones que pueden encontrarse en estos enfermos (contusión cerebral, hemorragia subaracnoidea, hemorragia intraventricular, lesiones en cuerpo calloso, tronco, núcleos profundos y colecciones extraaxiales (subduralepidural)).

Durante el ingreso se recogieron los hallazgos encontrados en la TC de control, así como los cambios con respecto al TC inicial. Además, siguiendo el protocolo anteriormente expuesto, se practicó en todos los casos una RM craneal, dentro de los primeros 30 días tras el trauma. Esta RM fue evaluada de manera ciega por dos neurorradiólogos que ignoraban al resultado de la TC inicial y la situación clínica inicial del paciente. Se registraron la presencia de contusiones y de lesiones sugestivas de LAD, así como su localización y su carácter hemorrágico o no hemorrágico, 
combinando para ello la información procedente de diferentes secuencias. Además, se clasificaron los hallazgos en RM según la clasificación de las lesiones asociadas con LAD descrita por Adams y adaptada por Gentry y cols para los hallazgos en RM, que sigue una gradación centrípeta de la severidad de las lesiones ${ }^{1,10}$. Cuando la apreciación de los dos neurorradiólogos difería se adoptó un grado por consenso. La clasificación de Adams y cols., divide la LAD en tres grados:
Grado 1
Lesiones en sustancia blanca lobar
Grado 2
Lesiones en cuerpo calloso
Grado 3
Lesiones en porciones dorsolaterales del

\section{Análisis estadístico}

Se realiza un análisis descriptivo de las variables demográficas de la serie y hallazgos en imagen en TC y RM. Para el establecimiento del grado de concordancia entre los dos neurorradiólogos se usó el coeficiente kappa. Para la comparación de los hallazgos en TC y RM se realiza una descripción de las diferencias de frecuencias de dichos hallazgos en la serie global, así como una aproximación a la sensibilidad de cada prueba con respecto a los diferentes hallazgos (capacidad de identificación de contusiones, lesiones en cuerpo calloso, tronco y núcleos profundos y presencia de hemorragia subaracnoidea) usando como frecuencia global de cada lesión la suma de los casos en los que se identifican con cada prueba.

Para identificar los hallazgos relacionados con la presencia de LAD en nuestra muestra se ha realizado un estudio univariable de los diferentes factores usando la prueba de $\chi^{2}$ y calculando los odds ratios con respecto a la presencia de LAD. Para establecer la relación entre la alteración del nivel de conciencia y localización de las lesiones de LAD se realizó un estudio de correlaciones simples con Rho de Spearman al tratarse la GCS de una variable ordinal no continua; posteriormente mediante la prueba de $\chi^{2}$ se han intentado establecer las diferentes odds ratios para cada uno de los estratos de la subescala motora del GCS. Por último, se ha intentado establecer la existencia de una relación lineal entre los mismos mediante un test de linealidad asociado a la $\chi 2$.

Todos los análisis se han llevado a cabo mediante el programa estadístico SPSS, estableciendo como límite de significación $\mathrm{p}<0.05$.

\section{Resultados}

\section{Características demográficas de la muestra}

Del total de enfermos revisados, 100 enfermos cum-
Tabla 1

Características demográficas de 100 enfermos con TCE grave y moderado

$\begin{array}{lc}\text { Edad } & \begin{array}{l}\text { Media:33 } \\ \text { Rango:15-7 }\end{array} \\ \text { Sexo n (\%) } & 83(83 \%) \\ \text { Hombre } & 17(17 \%) \\ \text { Mujer } & \\ \text { Gravedad del TCE n (\%) } & \\ \text { TCE grave (GCS } \leq 7) & 63(63 \%) \\ \text { TCE moderado (GCS 8-14) } & 37(37 \%) \\ \text { Mecanismo n (\%) } & \\ \text {-Accidente coche } & \\ \text {-Moto/Bicicleta } & 57(57 \%) \\ \text {-Atropello } & 13(13 \%) \\ \text {-Precipitación } & 8(8 \%) \\ \text {-Otros (Agresión/Caida) } & 12(12 \%) \\ & 10(10 \%)\end{array}$

plían los criterios de inclusión y en ellos se había realizado RM craneal en el primer mes tras el traumatismo craneal. La edad media de la muestra es de 33 años, siendo la mayor parte de los enfermos varones (Tabla 1). La mayor parte de los enfermos (63\%) habían sufrido un trauma grave (GCS postresucitación $<7$ ). El mecanismo más frecuente fue el accidente de tráfico, siendo el automóvil el medio de transporte más frecuente.

En cuanto a los factores relacionados con la gravedad del traumatismo sufrido, se debe destacar que un 33\% de los pacientes presentaba una puntuación motora en el GCS postresucitación menor o igual a 3, el 20\% un trauma extracraneal grave asociado, el 23\% hipotensión o hipoxia sospechada o confirmada (shock), y un $23 \%$ neurodeterioro, siendo las causas más frecuente la hipertensión intracraneal y la aparición de nuevas lesiones en TAC de control en cinco casos respectivamente.

\section{Hallazgos en TC}

En todos los enfermos se realizó TC craneal en las primeras 24 horas, seguido de TC de control para verificar si aparecían nuevas lesiones o si cambiaban las ya existentes. Los hallazgos en la TAC inicial se clasificaron según la clasificación de Marshall y cols. ${ }^{19}$, siendo el patrón más frecuente el de tipo I y II (70\%). En siete casos se produjeron cambios en la TAC de control, apareciendo nuevas lesiones en cinco.

En la Tabla 2 se muestran los hallazgos en la TAC. Es de destacar la alta frecuencia de hemorragia subaracnoidea traumática en la muestra, un $48 \%$ de los casos la presentaban, que, con mayor frecuencia, se dispone corticalmente. 
Tabla 2

\section{Hallazgos en TAC inicial}

Clasificación Marshall
Tipo I
Tipo II
Tipo III
Tipo IV
Tipo V
Hematoma subdural
Hematoma epidural
Contusion
Localización
-Frontal unilateral
-Bifrontal
-Temporal
-Bitemporal
-Otra

Lesión cuerpo calloso

Lesión núcleos profundos

Lesión en tronco

Lesión en cerebelo

Presencia de HSA

-Localización predominante:

+ Cortical

+Basal

$7(15 \%)$

+ Tentorial

$2(4 \%)$

\section{-Cantidad \\ + Trazas \\ +Abundante}

34

14

Presencia de HIV

$23(23 \%)$

Aparecieron contusiones en el 53\% de los casos siendo la localización más frecuente a nivel frontal y temporal. En 8 casos se detectaron lesiones en el cuerpo calloso y en 4 se identificaron lesiones en el tronco.

\section{Hallazgos en RM}

En todos los casos se realizó estudio RM, según la técnica descrita anteriormente, dentro de los primeros 30 días tras el traumatismo, con una mediana de días tras el mismo de 15 días. Para poder detectar el mayor número

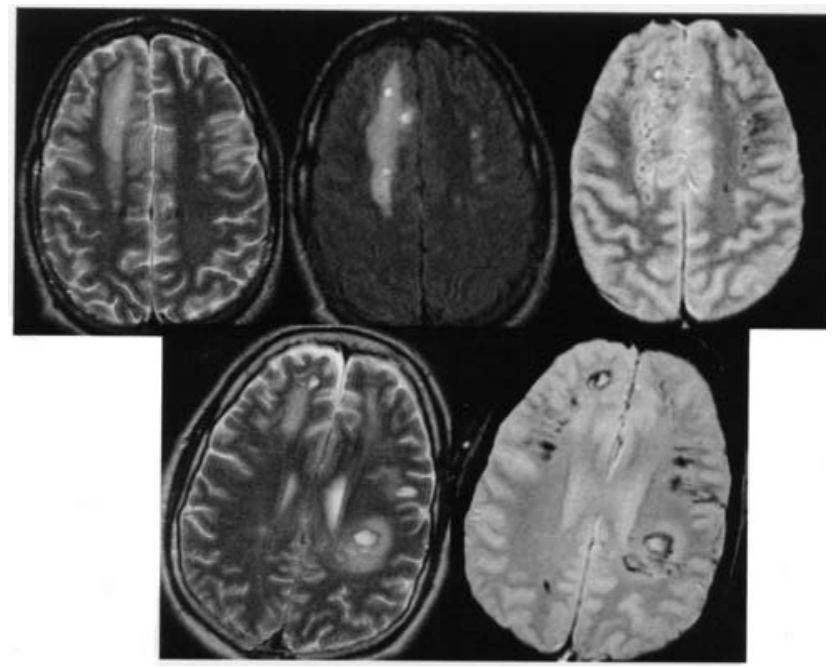

Figura 2. Lesiones en sustancia blanca subcortical en diferentes imágenes, T2 (izquierda arriba y abajo), Flair (centro arriba) y eco de gradiente (derecha arriba y abajo).

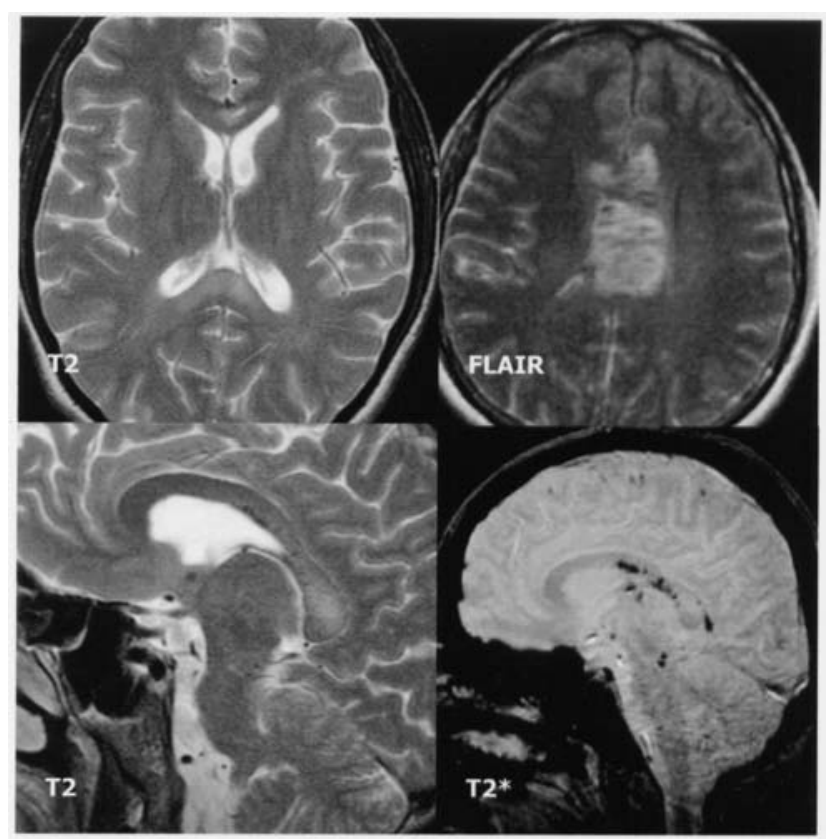

Figura 3. Lesiones en cuerpo calloso afectando esplenio (izquierda) y esplenio y cuerpo (derecha).

de lesiones se combinaron los hallazgos en las diferentes secuencias. Los hallazgos en dichos estudios quedan reflejados en la Tabla 3. Se detectaron contusiones en un 64\% de los individuos con una localización predominantemente frontal y temporal. Se detectaron signos de lesión axonal difusa en un $65 \%$ de los enfermos, siendo la gran mayoría de las lesiones $(81 \%)$ hemorrágicas, en tanto que las lesiones correspondientes a LAD no hemorrágica sólo se observaron en el 19\%. En 33 enfermos se detectaron lesiones 
Tabla 3

Hallazgos en RM (1)

Día de realización de RM

Contusiones

-Localización:

+Frontal unilateral

+ Bifrontal

+Temporal

+Bitemporal

+ Otra

Lesión axonal difusa

Localización

+Cápsula interna

+Cápsula externa

+Cuerpo calloso

+ Frontal

+Temporal

+Parietal

+Occipital

+Varios lóbulos

+ Periventricular

+ Cerebelo

+ Tronco

Proporcion hemorrágica/no hemorrágica

Núcleos profundos

HSA
Mediana:15 días

Rango intercuartílico: 6-20 días

$64(64 \%)$
$15(23 \%)$
$26(41 \%)$
$9(14 \%)$
$5(8 \%)$
$8(13 \%)$

$N(\%)$

$65(65 \%)$

Hemorrágica

No hemorrágica

10

6

36

55

38

6

3

39

11

4

33

202

$23(23 \%)$

$23(23 \%)$

$\begin{array}{rr}6 & 4 \\ 5 & 1 \\ 30 & 6 \\ 50 & 5 \\ 34 & 4 \\ 6 & 0 \\ 3 & 0\end{array}$

$6 \quad 5$

40

$20 \quad 13$

$164(81 \%) \quad 38(19 \%)$

14

9

4

1

6

5

4

0

(1)

4

\section{9}

Tabla 4

Hallazgos en RM (2). Lesiones en RM en cuerpo calloso y tronco

Lesión en cuerpo calloso

-Lesión en sustancia blanca

-Extensión:

+ Rodilla

+Cuerpo

+ Esplenio

+ Varias

Lesión en tronco

-Lesión en sustancia blanca

-Lesión en cuerpo calloso

-Lesión bilateral

-Localización:

+Mesencéfalo

+Protuberancia

+ Bulbo

+Varias
$36(36 \%)$

35

0

6

15

15

$33(33 \%)$

$31(94 \%)$

$23(70 \%)$

9

N

27

2

1

3 


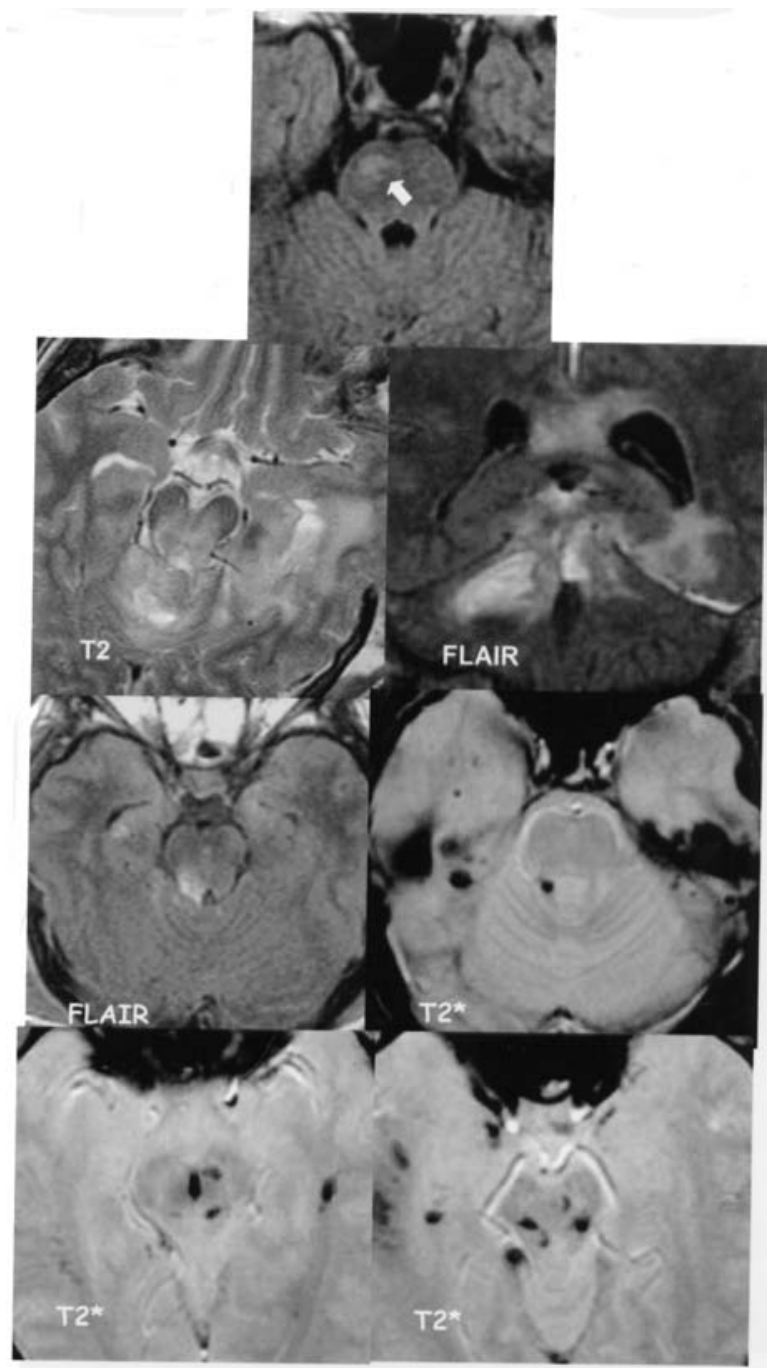

Figura 4. Lesiones en tronco. Arriba contusión anterior (flecha). Medio cuatro figuras mostrando lesiones dorsolaterales del mesencéfalo. Abajo LAD en forma de hemorragias petequiales en mesencéfalo.

que afectaban a varios lóbulos cerebrales. Un $23 \%$ de los enfermos presentaba lesiones en núcleos profundos, siendo éstas también en su mayoría hemorrágicas.

En 36 enfermos se detectaron lesiones en el cuerpo calloso, que con mayor frecuencia se distribuyeron a nivel del esplenio o afectando a varias zonas del cuerpo calloso (Tabla 4). La mayor parte de las lesiones también se definieron como hemorrágicas. Es de destacar que la mayor parte de los enfermos que presentaban lesiones en el cuerpo calloso también presentaron lesiones sugestivas de LAD a nivel de la sustancia blanca hemisférica.

Se detectaron lesiones en tronco en 33 enfermos. La mayor parte afectaron al mesencéfalo, bien anterior o en la parte posterior, probablemente como consecuencia de laceración del mesencéfalo contra el borde libre tentorial.

\begin{tabular}{lcc}
\hline \multicolumn{3}{c}{ Tabla 5 } \\
\multicolumn{1}{c}{ Comparación hallazgos en TAC y RM } \\
\hline & $N(\%)$ & Diferencia \\
Contusiones TAC & $53(53 \%)$ & \\
Contusiones RM & $64(64 \%)$ & $17 \%$ \\
Lesiones cuerpo calloso TAC & $8(8 \%)$ & \\
Lesiones cuerpo calloso RM & $36(36 \%)$ & $77 \%$ \\
& $4(4 \%)$ & \\
Lesiones tronco TAC & $33(33 \%)$ & $87 \%$ \\
Lesiones tronco RM & $10(10 \%)$ \\
Lesión núcleos profundos TAC & \\
Lesión núcleos profundos RM & $23(23 \%)$ & $57 \%$ \\
& $48(48 \%)$ & $52 \%$ \\
Presencia HSA TAC & $23(23 \%)$ & \\
Presencia HSA RM &
\end{tabular}

\section{Tabla 6}

Sensibilidad de la TC y RM a la identificación de diferentes lesiones. Sensibilidad $=$ Verdaderos positivos $/ \mathbf{N}$ total

\begin{tabular}{lr}
\hline Presencia de contusiones & $\mathrm{N}=73$ \\
TC & $53 / 73=73 \%$ \\
RM & $64 / 73=88 \%$ \\
& $\mathrm{~N}=36$ \\
Lesiones en cuerpo calloso & $8 / 36=22 \%$ \\
TC & $36 / 36=100 \%$ \\
RM & $\mathrm{N}=33$ \\
Lesiones en tronco & $4 / 33=12 \%$ \\
TC & $33 / 33=100 \%$ \\
RM & \\
& $\mathrm{N}=23$ \\
Lesiones en núcleos profundos & $10 / 23=43 \%$ \\
TC & $23 / 23=100 \%$ \\
RM & $\mathrm{N}=51$ \\
& \\
Presencia de HSA & $48 / 51=94 \%$ \\
TC & $23 / 51=45 \%$ \\
RM &
\end{tabular}

La gran mayoría presentaba lesiones sugestivas de LAD en sustancia blanca hemisférica, pero 10 de ellos no presentaban lesiones asociadas en el cuerpo calloso, siendo la distribución de las lesiones en tronco en estos casos similar a la del grupo general de lesiones de tronco.

Se clasificaron los hallazgos según las clasificaciones de Adams como se muestra en la Figura 1. Las RM fueron evaluadas de manera independiente y ciega por dos neurorradiólogos que desconocían la situación clínica del 

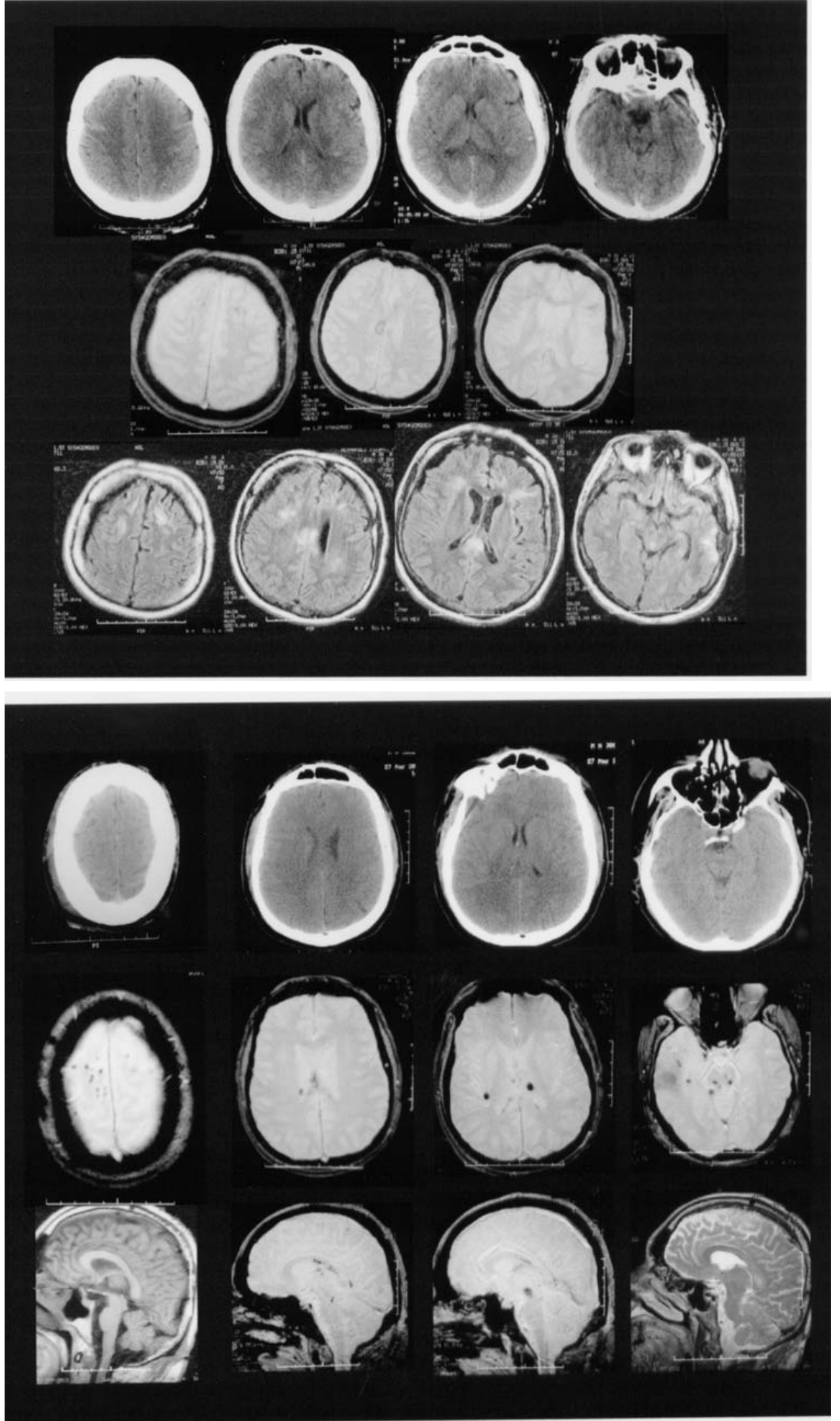

Figura 5. Enfermo de 38 años que sufrió TCE grave $(G C S=4, \quad G C S$ motor $=2)$, secundario a accidente de tráfico (coche). El TC inicial (arriba) se clasifica como tipo II según la clasificación de Marshall identificándose sólo hemorragia subaracnoidea en surcos de la convexidad frontal y temporal y fractura temporal izquierda. En RM eco de gradiente (centro) se observan pequeñas lesiones petequiales en sustancia blanca subcortical (izquierda), cuerpo calloso (centro) y sustancia blanca frontal (derecha). La secuencia flair (abajo) revela lesiones subcorticales frontales bilaterales, en cuerpo calloso (esplenio y cuerpo) y contusión temporal izquierda.

Figura 6. Varón de 42 años que sufre TCE grave ( $G C S=4$, GCS motor $=2)$, secundario a accidente de tráfico (coche). El TC inicial (arriba) se clasifica como tipo II según la clasificación de Marshall, identificándose pequeña hemorragia intraventricular. La $R M$ en eco de gradiente axial (centro) y sagital T1, eco de gradiente y T2, muestra lesiones hemorrágicas múltiples en sustancia blanca, cuerpo calloso y tronco. 
Tabla 7

Relación mecanismo del trauma y presencia de LAD en RM

\begin{tabular}{lrrr} 
Mecanismo & \multicolumn{1}{l}{$\underline{L A D}$} & $\underline{N o L A D}$ & OR (IC 95\%) \\
- Precipitación & $2(17 \%)$ & $10(83 \%)$ & 1 \\
- Otros (caída, agresión) & $4(40 \%)$ & $6(60 \%)$ & $3(0,5-24)$ \\
- Atropello & $4(50 \%)$ & $4(50 \%)$ & $5(0,6-39)$ \\
- Moto/bici & $8(62 \%)$ & $5(35 \%)$ & $8(1,2-53)$ \\
- Coche & $47(83 \%)$ & $10(17 \%)$ & $23(4-128)$
\end{tabular}

Tabla 8

Relación hallazgos en imagen relacionados con LAD en RM

$\underline{\text { LAD }} \quad \underline{\text { No LAD }} \quad \underline{\text { OR (IC 95\%) }}$

Presencia de HSA

$\begin{array}{lccc}\text { No } & 33(67 \%) & 16(33 \%) & \\ \mathrm{Si} & 32(63 \%) & 19(37 \%) & \\ & & & \\ \text { ia de Hemorragia intraventricular } & 43(56 \%) & 34(44 \%) & 17(2-135) \\ \mathrm{No} & 22(96 \%) & 1(4 \%) & \\ \mathrm{Si} & & & \\ & & 5(18 \%) & 0,3(0,1-0,9) \\ \text { No de contusiones } & 22(82 \%) & 30(41 \%) & \\ \text { Sí } & 43(59 \%) & \end{array}$

enfermo y el pronóstico del mismo. Cuando existieron diferencias entre los dos observadores se clasificaron los hallazgos por consenso. AI analizar el grado de coincidencia interobservador mediante el índice Kappa se comprobó que esta clasificación mostró una gran reproducibilidad interobservador (kappa de 0,96). Ejemplos de lesiones en sustancia blanca, cuerpo calloso y tronco se muestran en las Figuras 2, 3 y 4.

\section{Comparación hallazgos TC y RM}

Como se observa en las anteriores tablas y en la Tablas 5 y 6, la RM es más sensible que la TC para las lesiones en sustancia blanca cerebral, cuerpo calloso y tronco. Además detecta mayor número de contusiones. Sin embargo, parece menos sensible para la HSA traumática, no siendo capaz de detectaría hasta en un $52 \%$ de los enfermos que la presentaban en la TC inicial. Este hecho puede ser debido al tiempo transcurrido entre la TC inicial y la realización de la RM craneal. En las Figuras 5 y 6 se muestran casos en los que la TC no fue capaz de detectar lesiones que gracias a las diferentes secuencias de RM utilizadas fueron identificadas.

\section{Factores relacionados con la presencia de LAD en RM}

Se ha intentado establecer cuáles son los factores que están relacionados con la aparición de LAD en los enfermos de nuestra serie. Para ello se ha realizado un estudio univariable cruzando características clínicas y radiológicas que podrían estar relacionadas con estas lesiones.

Entre las características clínicas la más notable ha sido la asociación de la presencia de LAD y el mecanismo de producción del trauma. Parece que la LAD aparece más frecuentemente en traumas de mayor energía, sobre todo en los accidentes de tráfico, bien sea con automóvil o moto/ bici. El riesgo de presentar LAD en un accidente de tráfico es hasta 23 veces mayor que el riesgo de presentar LAD tras una precipitación (Tabla 7).

En cuanto a las características radiológicas asociadas a LAD la más claramente relacionada es la hemorragia intraventricular. La hemorragia intraventricular sobre todo se asocia a lesiones en el cuerpo calloso, ya que el $96 \%$ de los enfermos que presentaron hemorragia intraventricular presentaron lesiones en cuerpo calloso en RM, lo cual pone en evidencia que la hemorragia intraventricular probablemente sea secundaria al desgarro de pequeños vasos 
Tabla 9

Correlación entre lesiones diagnosticadas en RM y GCS postresucitación y GCS motor. Coeficientes de correlación Rho de Spearman

\begin{tabular}{|c|c|c|}
\hline & $\underline{G C S}$ & $\underline{\text { GCS motor }}$ \\
\hline Presencia de contusiones &, 1 & ,2 \\
\hline LAD en RM &,$- 45^{*}$ &,$- 45^{*}$ \\
\hline Lesión LAD sustancia blanca &,$- 42^{*}$ &,$- 42 *$ \\
\hline Lesión cuerpo calloso &,$- 31 *$ &,$- 34 *$ \\
\hline Lesión en tronco &,$- 41^{*}$ &,$- 39 *$ \\
\hline Clasificación LAD Adams &,$- 5^{*}$ &,$- 5^{* *}$ \\
\hline
\end{tabular}

producido por la lesión del cuerpo calloso como ya han apuntado algunos autores ${ }^{6}$. La hemorragia subaracnoidea traumática no se asocia a una mayor frecuencia de LAD detectada en RM, y las contusiones podrían tener un cierto efecto protector con respecto a la $\mathrm{LAD}$, lo cual se explica probablemente por el diferente mecanismo de producción de ambas lesiones (Tabla 8).

\section{Deterioro del nivel de conciencia tras TCE y profundidad de la $L A D$}

Según el modelo de Ommaya ${ }^{22}$, el trastorno de la conciencia tras el trauma sería proporcional a la energía del traumatismo y secundaria a la lesión cerebral, afectando esta a estructuras más profundas a medida que la energía fuera mayor. La lesión de estructuras cada vez más profundas, según un gradiente centrípeto desde la convexidad hasta el tronco, sería responsable del peor nivel de conciencia gradual de los enfermos expuestos a traumas de mayor energía.

Uno de los objetivos de nuestro estudio ha sido intentar establecer si existe una relación entre la profundidad de las lesiones de LAD en RM y el nivel de conciencia postresucitación. En nuestra serie existe una correlación negativa entre la presencia de lesiones asociadas a LAD, lesión en sustancia blanca hemisférica, lesión de tronco y la clasificación según el modelo centrípeto de estos hallazgos en la clasificaciones de Adams y el GCS postresucitación y la subescala motora del GCS (Tabla 9). Es decir, la presencia de daño cada vez más profundo y mayor puntuación en la escala de Adams se asocia a menor puntuación en la GCS y GCS motora y, por consiguiente, a peor nivel de conciencia.

De nuevo, la presencia de contusiones parece ir asociada a un trauma de menor energía y, por ello, se correlaciona débilmente, pero de forma positiva con el GCS inicial.

$\mathrm{Si}$ determinamos el riesgo de presentar lesiones asociadas LAD en RM según el GCS motor inicial, se puede observar que dicho riesgo aumenta a medida que disminuye el GCS motor inicial, existiendo una asociación linear entre intervalos en cada GCS motor y el riesgo de LAD, lesión en sustancia blanca, lesión en tronco y contusiones (Tabla 10). Estos resultados no se ven afectados si excluimos del análisis a los enfermos con lesión tipo $\mathrm{V}$ según la clasificación de Marshall, es decir, aquellos enfermos con efecto de masa en la TAC inicial. Parece pues clara la relación entre el peor nivel de conciencia inicial y la presencia de lesiones cada vez más profundas en RM, confirmando el modelo de Ommaya y Genarelli.

\section{Discusión}

\section{Métodos de imagen en el TCE}

En el contexto clínico del TCE y su manejo agudo, un método diagnóstico de imagen debe reunir una serie de

Tabla 10

Riesgo de presentar lesiones en RM según GCS motor

\begin{tabular}{cccccc} 
GCS motor & Contusiones & $\underline{\text { LAD }}$ & $\underline{\text { LAD sust blanca }}$ & $\underline{\text { LAD c. calloso }}$ & $\underline{\text { LAD tronco }}$ \\
\cline { 2 - 4 } 6 & 1 & 1 & 1 & 1 & 1 \\
5 & $0,7(0,2-3)$ & $6,8(1,9-24)$ & $6,4(1,7-23)$ & $10(1,2-91)$ & $1,3(1,1-1,7)$ \\
4 & $1,1(0,2-5,4)$ & $8,8(2,1-37)$ & $6,8(1,7-28)$ & $13(1,4-122)$ & $2(1,3-3)$ \\
3 & $0,2(0,1-1,5)$ & $27(2,7-272)$ & $27(2,7-272)$ & $26(2,3-288)$ & $3(1,2-7,5)$ \\
2 & $0,2(0,1-1,6)$ & $27(2,7-272)$ & $27(2,7-272)$ & $73(6-939)$ & $1,8(1,1-3,4)$ \\
1 & $0,2(0,1-1,4)$ & $22(3,6-132)$ & $12(2,4-63)$ & $14(1,4-133)$ & $1,8(1,1-3,4)$ \\
Linealidad $(\mathrm{p}<)$ & 0,05 & 0,01 & 0,01 & 0,01 & 0,01 \\
\hline
\end{tabular}


requisitos ${ }^{20}$ :

- Debe estar disponible en o cerca de la sala de emergencia o UCI que trate al enfermo.

- Debe poder ser utilizado de forma segura y fácil en un enfermo crítico.

- Su sensibilidad debe ser suficiente para determinar la gravedad, tipo y localización anatómica de la lesión: capacidad pronóstica.

- Debe ser suficientemente específico para determinar la categoría de la lesión (quirúrgico / no quirúrgico) para dirigir el tratamiento.

La introducción de la TC revolucionó el manejo del TCE, ya que es un método de imagen rápido y fácil de realizar, se puede realizar de forma segura a enfermos intubados/ventilados al aceptar todo tipo de materiales y es adecuado para guiar el tratamiento de estos enfermos, ya que permite una evaluación rápida y con buena definición de los sangrados y fracturas craneales.

La TC craneal ha demostrado su utilidad en la valoración de la patología potencialmente quirúrgica en el momento del trauma. Su utilización ha favorecido el mejor conocimiento de los mecanismos de la lesión traumática cerebral y ha mejorado el cuidado y tratamiento de los enfermos, reduciendo con su utilización la morbilidad y mortalidad de esta patología ${ }^{28}$.

Numerosos estudios han correlacionado la evolución final de los enfermos con numerosos parámetros anatómicos en relación con la gravedad de la lesión, tales como la presencia y tipo de lesión intracraneal ${ }^{16,17}$, la presencia de lesiones masa quirúrgica ${ }^{14}$, la desviación de línea media y la compresión de las cisternas basales o del tercer ventrículo $^{7,8}$.

En el momento actual, la clasificación de los hallazgos en TC más utilizada durante la etapa aguda-subaguda de la lesión es la clasificación del Traumatic Coma Data Bank (TCDB). Esta clasificación fue propuesta por Marshall y cols. y se basa en la situación de las cisternas mesencefálicas, la desviación de la línea media y la presencia o ausencia de lesiones focales para categorizar los hallazgos en TC en seis grupos diferentes ${ }^{19}$. Esta clasificación permite la identificación de sujetos en riesgo de sufrir deterioro secundario a hipertensión intracraneal. Además, permite el establecimiento del pronóstico de los enfermos en cuanto al riesgo de muerte, así como en categorías generales de buena y mala evolución. Sin embargo, no se ha demostrado su utilidad en cuanto a su relación con una determinación pronóstica más específica ni en cuanto a la capacidad de predecir alteraciones neuropsicológicas o trastornos neuropsiquiátricos en estos enfermos.

La TC craneal además presenta ciertas limitaciones a la hora de evaluar enfermos que han sufrido TCE. Por un lado es poco sensible a la hora de identificar la LAD, observándose un importante número de enfermos con discrepancias entre los hallazgos TC, que puede ser hasta normal, y una mala situación neurológica ${ }^{18}$. Es el caso de la lesión difusa tipo I y II según el TCDB, en la que la lesión difusa es definida de forma negativa, es decir, como presencia de coma sin efecto de masa. Además, es poco sensible a lesiones a nivel de la fosa posterior y en especial a nivel del tronco, cuya presencia nos indicaría la presencia de la LAD más grave. También es poco sensible a lesiones de LAD no hemorrágicas.

La RM es una técnica muy sensible a los cambios de la sustancia blanca y lesiones en fosa posterior, por ello, teóricamente, sería muy útil para detectar la LAD. Sin embargo, su utilidad en el momento agudo del trauma se ve dificultada por la duración de las exploraciones en enfermos inestables y la incompatibilidad de determinados materiales con la RM (tubos intubación, respiradores...). No obstante, estas dificultades teóricas están siendo superadas por el uso de materiales compatibles con la RM y la menor duración de las exploraciones usando la maquinaria disponible en la actualidad.

Determinadas secuencias de RM son muy sensibles a la detección de lesiones de LAD, sobre todo aquellas con tiempos de relajación y emisión largos. Las secuencias T2 son útiles, pero tienen limitaciones en lesiones periventriculares o corticales debido a la presencia de líquido cefalorraquídeo (LCR) próximo. Las secuencias FLAIR (Fluid attenuated inversion recovery) reducen o anulan la señal del líquido detectando un mayor número de lesiones. Las secuencias eco de gradiente en T2 son muy sensibles a la presencia de sangre o sus productos de degradación, siendo muy sensible a las lesiones de LAD hemorrágico, sobre todo si pasa algún tiempo desde el trauma hasta la realización de la RM. Nuestro trabajo muestra una mayor proporción de lesiones relacionadas con LAD hemorrágicas que en otros trabajos recientemente publicados ${ }^{3}$. Esto puede ser debido a la inclusión de secuencias de echo de gradiente que no son usadas por otros autores. Por ello, la evaluación con RM de los enfermos que han sufrido TCE se basa en el uso de estas tres secuencias, que son complementarias, usando además diversos planos de corte.

Diferentes autores han mostrado una mayor sensibilidad de la RM sobre la TC a la hora de diagnosticar el sustrato anatómico del daño cerebral traumático. Gentry y cols. en el año 1987, en una serie de 40 enfermos con TCE grave estudiados con TC craneal y RM precoz, demostraron una mayor sensibilidad de la RM frente a la TC sobre todo a nivel de lesiones no hemorrágicas, detectadas con mayor sensibilidad por RM con secuencias en T2, y lesiones en tronco cerebral ${ }^{11}$. Willberger y cols. estudiaron 24 enfermos con TCE grave, con y sin lesiones en $\mathrm{TC}^{30}$. En todos ellos existían lesiones en RM y además en 5 de ellos existían lesiones en el tronco que en ningún caso habían sido diagnosticadas mediante TC. Existen otros trabajos 
con hallazgos similares ${ }^{13,21,23,31}$.

En nuestra experiencia es evidente la mayor sensibilidad de la RM en la detección de lesiones tales como contusiones, lesiones en cuerpo calloso, núcleos profundos y tronco. Esta mayor sensibilidad a la hora de detectar lesiones que van a tener un importante peso en el pronóstico de estos enfermos tales como las lesiones de tronco hacen de la RM una técnica necesaria para el correcto establecimiento del pronóstico de los enfermos con TCE grave y moderado.

\section{Tipos de lesiones en el TCE}

De acuerdo con los trabajos de Adams y cols. ${ }^{1,2}$, el daño cerebral debido al TCE se divide en lesiones primarias y secundarias. La lesión primaria es el resultado de fuerzas mecánicas que producen deformación de los tejidos en el momento del impacto. Estas deformaciones dañarían directamente vasos sanguíneos, axones, neuronas y glia. Este daño primario iniciaría toda una serie de eventos inflamatorios, neuroquímicos y metabólicos que determinarían mayor daño cerebral, y que se clasifican como lesiones secundarias. Los diferentes tipos de lesiones secundarias son potencialmente reversibles con tratamiento adecuado y si son reconocidas a tiempo, por lo menos desde el punto de vista teórico.

Una aproximación diferente a las lesiones producidas por el TCE fue introducido por Gennarelli y cols ${ }^{9,22,25}$. Basándose en hallazgos clínico-radiológicos y neuropatológicos estos autores clasificaron las lesiones primarias en lesiones focales y difusas. Las lesiones focales serán aquellas que serían tan grandes como para ser vistas a simple vista. Estas lesiones producirían disfunción neurológica debido a una alteración local y sólo producirían alteraciones del nivel de conciencia o coma cuando fueran tan grandes como para provocar hipertensión intracraneal, herniación cerebral y/o compresión del tronco cerebral. Dentro de estas lesiones focales se incluyen las contusiones, hematoma subdural, epidural e intracerebral. Sin embargo, las lesiones difusas no se caracterizan por ser lesiones macroscópicamente localizadas o visibles significativamente en principio, sino que los pacientes que las presentan tienen una disfunción global de la función cerebral, pudiendo sufrir alteración del nivel de conciencia y coma sin necesidad de presentar hipertensión intracraneal, lesión masa o compresión del tronco cerebral. Muchos autores incluyen dentro de la lesión difusa la lesión axonal difusa, el daño isquémico cerebral secundario a hipoxia así como el edema cerebral difuso. La lesión más importante, por ser una lesión primaria dentro de este grupo, es la lesión difusa de la sustancia blanca denominada lesión axonal difusa.

Estos dos tipos morfológicos de lesión vendrían producidos por las características del trauma inicial y las fuerzas mecánicas que se dieran en ese momento. Cuando la cabeza sufre un cambio brusco de dirección se pueden inducir dos tipos de aceleración: translación y rotación. En general translación se refiere al movimiento de la cabeza en un solo eje o en una línea recta, mientras que rotación se refiere a que la cabeza gira en torno a su centro de gravedad o se mueve en varios ejes. Ommaya y Gennarelli observaron en modelos experimentales con primates que eran sometidos a diferentes tipos de aceleración/deceleración, cómo estos dos tipos de lesión se producían ante diferentes tipos de movimientos $^{22}$. Así, las contusiones y otras lesiones focales se producían en sujetos expuestos a aceleraciones en un plano, y los primates no sufrían alteración del nivel de conciencia en ausencia de contusiones en crecimiento. Sin embargo, si se permitía el movimiento en varias direcciones con aceleración/deceleración rotacional, se producían alteraciones del nivel de conciencia en ausencia de lesiones expansivas. En estos casos, el examen neuropatológico permitía la observación de los hallazgos característicos de la lesión axonal difusa (LAD) ${ }^{26}$.

Esta diferencia en el mecanismo de producción de las lesiones focales y difusas también es extrapolable al humano, ya que en general, en los traumatismos en los que se producen impactos directos, de corta duración sin movimientos rotacionales de la cabeza, como en las caídas o golpes directos predominan las contusiones o hematomas, mientras que en los traumatismos en los que predominan las aceleraciones/deceleraciones de duración moderada y en varios ejes, como ocurre en los accidentes de tráfico, la LAD es frecuente.

Como se muestra en nuestro trabajo, la presencia de LAD, definida por la prueba que con mayor sensibilidad la detecta, la RM, está en relación al mecanismo causal del traumatismo, siendo más frecuente su presencia cuando el mecanismo es de alta energía y el enfermo está sujeto a fuerzas de aceleración/deceleración importantes como son los accidentes de tráfico, con coche, moto/bici o atropello. Sin embargo, aquellos traumatismos en los que incide más el impacto directo, tales como los causados por precipitación, caída o agresiones directas tienen menor frecuencia y menor riesgo relativo de presentar LAD en RM. En estos traumatismos la frecuencia de contusiones es mayor $(60 \%$ en tráficos frente a $85 \%$ en precipitaciones y traumas directos), lo cual explica el factor protector de las contusiones para la presencia de LAD.

\section{Lesión axonal difusa: teoría centrípeta de Ommaya. Gra- dación de la $L A D$}

Strich (1956) fue el primer autor en encontrar en una serie necrópsica la aparición de una "degeneración difusa de la sustancia blanca" en una serie de enfermos con demencia postraumática severa ${ }^{27}$. Afirmó que el daño era 
producido por la torsión de los axones en el momento inicial del trauma. Más tarde Gennarelli y Adams acuñaron el término lesión axonal difusa para describir los hallazgos neuropatológicos de la lesión más devastadora que puede ocurrir tras el trauma ${ }^{1,22}$. De acuerdo con Povlishock y Cristman, la lesión axonal difusa puede definirse como la destrucción dispersa de axones a lo largo de los cerebros de animales y humanos que han sufrido una lesión traumática cerebral, en la que se ha implicado, de forma característica, la aceleración/deceleración de la cabeza ${ }^{24}$.

El daño axonal se produciría ante una aceleración/ deceleración por la fijación de algunas estructuras cerebrales, por el movimiento diferencial de estructuras superficiales y profundas o por la diferente consistencia, densidad y composición de diferentes partes del encéfalo que determinan diferentes efectos de la aceleración. Así, las lesiones se localizan con mayor frecuencia a nivel de la unión sustancia blanca/sustancia gris y en la sustancia blanca central profunda (cuerpo calloso, región periventricular, parahipocámpica, pedúnculos cerebrales y sustancia reticular ascendente).

La gravedad y localización de la lesión axonal difusa varía con la gravedad del trauma, tanto en el modelo experimental de Ommaya y Genarelli como en el humano, según la teoría centrípeta de Ommaya ${ }^{22}$. Así, este autor observó que la distribución de las tensiones lesivas inducidas por la inercia disminuirían en magnitud desde la superficie al centro aproximado de la casi esferoidal masa cerebral. De esta manera, se producen una gradación de síndromes clínicos tras el TCE, donde una mayor gravedad de la alteración del nivel y los contenidos de la conciencia son causadas por lesiones cada vez más profundas o centrales en el cerebro. De acuerdo con esta teoría, el tronco cerebral y el mesencéfalo serían las últimas estructuras en ser afectadas, tanto funcional como estructuralmente, y siempre que se observe lesión en estas estructuras, debería observarse lesión en estructuras más superficiales, tales como la sustancia blanca cerebral o el cuerpo calloso.

En nuestro trabajo se replica en términos generales el modelo de Ommaya, ya que existe una relación clara y lineal entre la alteración del nivel de conciencia postresucitación y la presencia y profundidad de las lesiones relacionadas con LAD. Además, podemos afirmar que la clasificación de Adams y cols. también es compatible con lo observado en nuestra serie, ya que los enfermos con lesiones en zonas más profundas suelen presentar lesiones en las estaciones anteriores.

\section{Conclusiones}

A pesar de ser una serie seleccionada, ya que la mayor parte de las RMs se realizaron fuera de la UCI y por ello los enfermos de nuestra serie son enfermos que han sobre- vivido la fase inicial del trauma, podemos decir, al menos que en los enfermos de nuestra serie:

-La RM es una técnica con mayor capacidad de detección de lesiones traumáticas que la TAC.

-Existe una relación evidente entre la localización en profundidad de las lesiones en la RM y la gravedad del traumatismo expresada por la escala de coma de Glasgow y su subescala motora.

-Se confirma el modelo de Ommaya en el que el mayor deterioro del nivel de conciencia está causado por una mayor profundidad de la lesión.

\section{Agradecimientos}

Este trabajo ha sido realizado gracias a una beca de la Fundación Mutua Madrileña Automovilística.

\section{Bibliografia}

1. Adams, J.H., Doyle, D., Ford, I., Gennarelli, T.A., Graham, D.I, McLellan, D.R.: Diffuse axonal injury in head injury: definition, diagnosis and grading. Histopathology 1989; 15: 49-59.

2. Adams, J.H., Graham, D.I., Gennarelli, T.A., Maxwell, W.L.: Diffuse axonal injury in non-missile head injury. J Neurol Neurosurg Psychiatry 1991; 54: 481-483.

3. Aguas, J., Begue, R., Díez, J.: [Brainstem injury diagnosed by MRI. An epidemiologic and prognostic reappraisal]. Neurocirugia 2005; 16: 14-20.

4. Boto, G.R., Gómez, P.A., De la, C.J., Lobato, R.D.: [Prognostic factors in severe head injury]. Neurocirugia 2004; 15: 233-247.

5. Boto, G.R., Gómez, P.A., De la, C.J., Lobato, R.D.: [Overview of the recent clinical trials in severe head injury and analysis of their therapeutic failure]. Neurocirugia 2005; 16: 39-49.

6. Cordobés, F., de la Fuente, M., Lobato, R.D.: Intraventricular hemorrhage in severe head injury. J Neurosurg 1983; 58: 217-222.

7. Cordobés, F., Lobato, R.D., Rivas, J.J., Cabrera, A., Sarabia, M., Castro, S., Cisneros, C., Torres, I.D., Lamas, E.: Post-traumatic diffuse axonal brain injury. Analysis of 78 patients studied with computed tomography. Acta Neurochir (Wien ) 1986; 81: 27-35.

8. Eisenberg, H.M., Gary, H.E. Jr., Aldrich, E.F., Saydjari, C., Turner, B., Foulkes, M.A., Jane, J.A., Marmarou, A., Marshall, L.F., Young, H.F.: Initial CT findings in 753 patients with severe head injury. A report from the NIH Traumatic Coma Data Bank. J Neurosurg 1990; 73: 688-698.

9. Gennarelli, T.A.: Animal models of human head injury. J Neurotrauma 1994; 11: 357-368.

10. Gentry, L.R., Godersky, J.C., Thompson, B.: MR imaging of head trauma: review of the distribution and radiopa- 
thologic features of traumatic lesions. AJR Am J Roentgenol 1988; 150: 663-672.

11. Gentry, L.R., Godersky, J.C., Thompson, B., Dunn, V.D.: Prospective comparative study of intermediate-field MR and $\mathrm{CT}$ in the evaluation of closed head trauma. AJR Am J Roentgenol 1988; 150: 673-682.

12. Hammoud, D., Wasserman, B.A.: Diffuse axonal injuries:pathophysiology and imaging. Neuroimag Clin N AM 2002; 12: 205-216.

13. Kelly, A.B., Zimmerman, R.D., Snow, R.B., Gandy, S.E., Heier, L.A., Deck, M.D.: Head trauma: comparison of MR and CT--experience in 100 patients. AJNR Am J Neuroradiol 1988; 9: 699-708.

14. Levati, A., Farina, M.L., Vecchi, G.: Prognosis of severe head injuries. J Neurosurg 1982; 57: 779-783.

15. Levin, H.S., Williams, D., Crofford, M.J., High, W.M. Jr., Eisenberg, H.M., Amparo, E.G., Guinto, F.C. Jr., Kalisky, Z., Handel, S.F., Goldman, A.M.: Relationship of depth of brain lesions to consciousness and outcome after closed head injury. J Neurosurg 1988; 69: 861-866.

16. Lobato, R.D., Cordobés, F., Rivas, J.J., de la F.M., Montero, A., Bárcena, A., Pérez, C., Cabrera, A, Lamas, E.: Outcome from severe head injury related to the type of intracranial lesion. A computerized tomography study. J Neurosurg 1983; 59: 762-774.

17. Lobato, R.D., Gómez, P.A., Alday, R., Rivas, J.J., Domínguez, J., Cabrera, A., Turanzas, F.S., Benítez, A., Rivero, B.: Sequential computerized tomography changes and related final outcome in severe head injury patients. Acta Neurochir (Wien ) 1997; 139: 385-391.

18. Lobato, R.D., Sarabia, R., Rivas, J.J., Cordobés, F., Castro, S., Muñoz, M.J., Cabrera, A., Bárcena, A., Lamas, E.: Normal computerized tomography scans in severe head injury. Prognostic and clinical management implications. J Neurosurg 1986; 65: 784-789.

19. Marshall, L.F., Klauber, M.R., Van Berkum, Clark, M., Eisenberg, H., Jane, J.A.: A new classification of head injury based on computerized tomography. J. Neurosurg. 1991; 75 (Suppl), 14-20.

20. Mataro, M., Poca, M.A., Sahuquillo, J., Pedraza, S., Ariza, M., Amorós, S., Junque, C.: Neuropsychological outcome in relation to the traumatic coma data bank classification of computed tomography imaging. J Neurotrauma 2001; 18 : 869-879.

21. Ogawa, T., Sekino, H., Uzura, M., Sakamoto, T., Taguchi, Y., Yamaguchi, Y., Hayashi, T., Yamanaka, I., Oohama, N., Imaki, S.: Comparative study of magnetic resonance and CT scan imaging in cases of severe head injury. Acta Neuro- chir Suppl (Wien ) 1992; 55: 8-10.

22. Ommaya, A.K. Gennarelli, T.A.: Cerebral concussion and traumatic unconsciousness. Correlation of experimental and clinical observations of blunt head injuries. Brain 1974; 97: 633-654.

23. Parizel, P.M., Ozsarlak, Van Goethem, J.W., Van Den, H.L., Dillen, C., Verlooy, J., Cosyns, P., De Schepper, A.M.: Imaging findings in diffuse axonal injury after closed head trauma. Eur Radiol 1998; 8: 960-965.

24. Povlishock, J.T. Christman, C.W.: The pathobiology of traumatically induced axonal injury in animals and humans: a review of current thoughts. J Neurotrauma 1995; 12: 555564.

25. Sahuquillo, J. Poca, M.A.: Diffuse axonal injury after head trauma. A review. Adv Tech Stand Neurosurg 2002; 27 : 23-86.

26. Sahuquillo, J., Vilalta-Castán, J., Lamarca, J., Rubio, E., Rodríguez-Pazos, M.: Diffuse axonal injury after severe head trauma. A clinico-pathological study. Acta Neurochir (Wien ) 1989; 101: 762-774.

27. Strich, S.: Diffuse degeneration of cerebral white matter in severe dementia following head injury. J Neurol Neurosurg Psychiatry 1956; 19: 163-185.

28. Teasdale, G., Galbraith, S., Murray, L.S.: Management of traumatic intracranial hematoma. BMJ 1983; 285: 16951697.

29. Vilalta-Castán, J., Sahuquillo, J., Rubio, E.: Traumatismos craneoencefálicos graves sin lesiones significativas en la TAC. Revista de Neurología 1984; 57; 133-140.

30. Wilberger, J.E., Deeb, Z., Rothfus, W.: Magnetic resonance imaging in cases of severe head injury. Neurosurgery 1987; 20: 571-576.

31. Zimmerman, R.A., Bilaniuk, L.T., Hackney, D.B., Goldberg, H.I., Grossman, R.I.: Head injury: early results of comparing CT and high-field MR. AJR Am J Roentgenol 1986; 147: 1215-1222.

Lagares, A.; Ramos, A,; Alday, R.; Ballenilla, F.; Pérez, A,; Arrese, I.; Fernández-Alén, J.A.; Pascual, B.; Kaen, A.; Gómez, P.A.; Lobato, R.D.: Resonancia magnética en trauma craneal moderado y grave: estudio comparativo de hallazgos en TC y RM. Características relacionadas con la presencia y localización de lesión axonal difusa en RM. Neurocirugía 2006; 17: 105-118.

Correspondencia postal: Alfonso Lagares, Servicio de Neurocirugía. Hospital 12 de Octubre. Crta de Andalucia km 5,4. 28041 Madrid. 